\title{
BIANCHI TYPE I COSMOLOGICAL MODELS WITH EXPANSION DRIVEN BY PARTICLE CREATION
}

\author{
Kalyani Desikan \\ Department of Mathematics, School of Advanced Sciences \\ Vellore Institute of Technology, Chennai-600127, India \\ Email: kalyanidesikan@vit.ac.in
}

\begin{abstract}
A study of Bianchi Type I cosmological model is undertaken in the framework of creation of particles. To accommodate the creation of new particles, the universe is regarded as an Open thermodynamical system. The energy conservation equation is modified with the incorporation of a creation pressure in the energy momentum tensor. Exact solutions of the field equations are obtained (i) for a particular choice of the particle creation function and (ii) by considering the deceleration parameter to be constant. In the first model the behavior of the solution at late times is investigated. The physical aspects of the model have also been discussed. In the case of the second model we have restricted our analysis to the power law behaviour for the average scale factor. This leads to a particular form for the particle creation function. The behavior of the solution is investigated and the physical aspects of the model have also been discussed for the matter dominated era.
\end{abstract}

Keywords: Cosmology - Bianchi Type I - Particle creation - Open thermodynamic system -Constant Deceleration Parameter - Accelerated expansion - Late time acceleration

\section{Introduction}

Anisotropic cosmological models play an important role in understanding the behaviour of the universe in its early stages of evolution. Cosmological observations support the existence of an anisotropic phase that approaches an isotropic one [1]. It is well known that the exact solutions of General Theory of Relativity for homogeneous space time belong to either Bianchi type or Kantowaski-Sachs space time. Bianchi type I cosmological models have been studied by a number of authors in different contexts [2-4]. Krori and Mukherjee [5] studied the evolution of Bianchi cosmologies with bulk viscosity and particle creation. Bhanja et al. [6] presented an anisotropic model of the universe with constant energy per particle. They considered a decaying cosmological constant and particle production in an adiabatic process as the sources for the entropy.

Since the discovery of the late-time accelerated expansion of the Universe [7, 8] various explanations are being provided to satisfactorily explain the phenomenon. Dark energy is considered by many to be the cause of the observed late-time acceleration of the universe. This dark energy dominates over matter and has a repulsive effect. Several candidates of dark energy are found in the literature. The most favoured candidate of dark energy, from the different cosmological observations, is the cosmological constant $\Lambda[9,10]$. Although explanations for the accelerated expansion in terms of scalar fields like quintessence [11], K-essence [12], phantom fields [13] and Chaplygin gas [14] are available, a number of models involving the cosmological term $\Lambda$, especially time-varying $\Lambda$ have also been proposed [15-21].
The classical evolution equations are purely adiabatic and reversible; consequently, they cannot provide by themselves an explanation for the origin of cosmological entropy which might have been generated through irreversible processes during the cosmic expansion. Prigogine et al. [22, 23] have investigated the role of irreversible processes in creation of matter out of gravitational energy in the context of General Relativity. It was shown by Prigogine and Ge'he'niau [24] and Prigogine and Glansdorff [25] that thermodynamics of open systems when applied to cosmology, leads to a reinterpretation in Einstein's equations of the matterenergy stress tensor [22]. Here the universe starts from a random vacuum fluctuation and the cosmic expansion is driven by the creation of matter particles. The effect of creation of new particles is equivalent to adding a supplementary negative pressure term to the thermodynamic pressure and this drives the expansion.

The results of Prigogine, Ge'he'niau, Gunzig and Nardone [22, 23] were further discussed and generalized by Calva o, Lima and Waga [26] through a covariant formulation allowing specific entropy variation as usually expected for nonequilibrium processes in fluids. Sudharsan and Johri [27] investigated the effect of bulk viscosity on the evolution of the FRW models in the context of open thermodynamic systems. The effect of bulk viscosity with a time varying bulk viscous coefficient in the framework of irreversible thermodynamics for homogeneous and isotropic space-times has been considered by Desikan [28]. By choosing appropriate functions for the particle creation rates these models lead to non-singular beginnings and inflationary behavior. In the framework of the FRW isotropic geometry Lima, Germano and Abramo 
[29] and Abramo and Lima [30] have considered inflationary cosmological models with matter creation. In these papers the authors obtained the conditions for inflation; they proposed a minimally modified hot big-bang model and examined the dynamical properties of a flat, inflationary universe with matter creation. Lima [31] analysed the problem of the compatibility of the continuous creation of photons and the present black-body nature of the cosmic microwave radiation. Using thermodynamic and semiclassical considerations, he derived a new Planckian type distribution for cosmologies with matter creation. The proposed spectrum is preserved during the evolution of the Universe and is compatible with the present spectral shape of the cosmic microwave background.

Johri and Desikan [32] extended the hypothesis of Prigogine, Ge'he'niau, Gunzig and Nardone [22,23] of creation of matter out of gravitational energy to Brans-Dicke type cosmological models. By incorporating the creation pressure in the energymomentum tensor, they obtained exact solutions of the field equations of Brans-Dicke theory by assuming a power law dependence of the scalar field on the scale factor. The resulting models have constant deceleration parameter. Singh and Beesham [33], Singh et al. [34] studied cosmological models having bulk viscosity and particle creation within the frame work of Brans-Dicke theory. Singh et al. [35] have also studied the effect of particle production in higher derivative theory.

Harko and Mak [36] have analyzed the effects of particle creation in inflationary cosmology in the context of the thermodynamics of open systems and obtained a generalization of the elementary reheating theory. Brevik and Stokkan [37] explored the question whether the viscosity / irreversible matter creation concepts describe the same physical process and came to the conclusion that they do not. Gunzig, Maartens and Nesteruk [38] developed a thermodynamically consistent cosmology with a phenomenological model of quantum creation of radiation due to vacuum decay considering a particle creation rate proportional to the total energy density. Zimdahl and Pavo'n [39], Zimdahl, Pavo'n and Jou [40], Zimdahl and Pavo'n [41] and Zimdahl and Pavo'n [42] proposed an alternative phenomenological view on matter creation processes in the early Universe and their influence on the dynamics of the space-time. In this approach in addition to the familiar relations for viscous cosmologies they added an explicit balance law for the particle number to the equations describing the dynamics of the early universe.

Bianchi type space- times play a vital role in understanding the early stages of evolution of the universe. In particular, the simplest of anisotropic models are Bianchi type-I homogeneous models whose spatial sections are flat, but the expansion or contraction rates are direction dependent. Many researchers [43-48] have investigated Bianchi type-I models with different points of view to understand the possible effects of anisotropy in the early Universe on present day observations. Harko and Mak [49] also studied the effect of matter creation on the evolution and dynamics of an anisotropic Bianchi type I space-time in the framework of open thermodynamic systems theory. They obtained the general solution in parametric form for a cosmological fluid obeying a Zel'dovich type equation of state $(\rho=p)$ and with particle creation rate proportional to the square of the mean Hubble function and to the energy density of matter, respectively.

Singh and Kale [50] investigated the role of particle creation and bulk viscosity on the evolution of homogeneous and anisotropic model of the universe represented by Bianchi type I space time metric. They considered particle creation and bulk viscosity as separated irreversible processes. In order to discuss physical and geometrical behaviour of the model, they obtained a new set of exact solutions of Einstein's field equation in non-causal, truncated and full causal theories.

Motivated by the above mentioned studies, in this paper, we attempt to apply the concept of creation of matter as a possible mechanism to explain the accelerated expansion of the universe in the context of Bianchi type I anisotropic model of the universe. The modified field equations in the presence of particle creation are given in section 2 . We see that the number of equations is less than the number of unknowns. Hence, we have looked at two different additional assumptions in order to make the system of equations well defined and enable us to obtain unique solutions. The first of the additional assumptions considered is with respect to the particle creation function $\mathrm{N}(\mathrm{t})$. In the second model, the deceleration parameter is taken to be a constant. Section 3 deals with the solutions of the field equations for the first model. Section 4 presents the solutions and discussions for the second model.

\section{Modified Einstein's Field Equations with Creation of Matter}

Let us consider the universe as an open thermodynamic system with $N$ particles. Suppose a random vacuum fluctuation induces a transformation of gravitational energy into matter energy, creating additional number of $d N$ particles. This gives rise to a negative pressure $p_{c}$. Hence, the effective energy-momentum tensor of the cosmic fluid in the presence of creation of matter includes the creation pressure term $p_{c}$ and is given by

$\tilde{T}_{a b}=\left(\rho+p+p_{c}\right) u_{a} u_{b}-\left(p+p_{c}\right) g_{a b}$ 
where $\rho$ and $p$ are the energy density and pressure, respectively, $u_{a}$ is the fluid-four velocity and $g_{a b}$ is the metric tensor. Accordingly, the modified Einstein gravitational field equations are given by

$$
G_{a b}=8 \pi G \tilde{T}_{a b}
$$

The line element of a Bianchi type I space-time, which generalizes the flat FRW metric to the anisotropic case is given by

$$
d s^{2}=d t^{2}-\left(R_{i}(t) d x^{i}\right)^{2}
$$

where $R_{i}(t), i=1,2,3$ are the scale factors.

The field equations (2) with the above metric and the barotropic equation of state

$$
p=\gamma \rho,-1 \leq \gamma \leq 1
$$

now give

$$
\begin{aligned}
& \frac{\dot{R}_{1} \dot{R}_{2}}{R_{1} R_{2}}+\frac{\dot{R}_{1} \dot{R}_{3}}{R_{1} R_{3}}+\frac{\dot{R}_{2} \dot{R}_{3}}{R_{2} R_{3}}=8 \pi G \rho \\
& 2 \frac{\ddot{R}_{1}}{R_{1}}+\frac{\dot{R}_{1} \dot{R}_{2}}{R_{1} R_{2}}+\frac{\dot{R}_{1} \dot{R}_{3}}{R_{1} R_{3}}-\frac{\dot{R}_{2} \dot{R}_{3}}{R_{2} R_{3}}=-8 \pi G\left(p+p_{c}\right) \\
& 2 \frac{\ddot{R}_{2}}{R_{2}}+\frac{\dot{R}_{1} \dot{R}_{2}}{R_{1} R_{2}}+\frac{\dot{R}_{2} \dot{R}_{3}}{R_{2} R_{3}}-\frac{\dot{R}_{1} \dot{R}_{3}}{R_{1} R_{3}}=-8 \pi G\left(p+p_{c}\right) \\
& 2 \frac{\ddot{R}_{3}}{R_{3}}+\frac{\dot{R}_{1} \dot{R}_{3}}{R_{1} R_{3}}+\frac{\dot{R}_{2} \dot{R}_{3}}{R_{2} R_{3}}-\frac{\dot{R}_{1} \dot{R}_{2}}{R_{1} R_{2}}=-8 \pi G\left(p+p_{c}\right)
\end{aligned}
$$

Equations (5-8) lead to the continuity equation

$$
\dot{\rho}+\left(\rho+p+p_{c}\right)\left(\frac{\dot{R}_{1}}{R_{1}}+\frac{\dot{R}_{2}}{R_{2}}+\frac{\dot{R}_{3}}{R_{3}}\right)=0
$$

with

$$
p_{c}=\frac{-(\rho+p)}{N} \frac{d N}{d V} V=-(1+\gamma) \rho \frac{\dot{N}}{N} \frac{V}{\dot{V}}
$$

Letting $\quad V=R_{1} R_{2} R_{3}$ (volume scale factor), equation (10) reduces to

$$
p_{c}=-(1+\gamma) \rho \frac{\dot{N}}{N}\left(\frac{\dot{R}_{1}}{R_{1}}+\frac{\dot{R}_{2}}{R_{2}}+\frac{\dot{R}_{3}}{R_{3}}\right)^{-1}
$$

Equation (11) can be rewritten as

$$
p_{c}=-(1+\gamma) \rho \frac{\dot{N}}{N} \frac{1}{3 H}
$$

where $3 H=\frac{\dot{R}_{1}}{R_{1}}+\frac{\dot{R}_{2}}{R_{2}}+\frac{\dot{R}_{3}}{R_{3}}$.
The cosmological models based on the above equations are more general, because they involve three cosmological parameters $\rho(t), p(t)$ and $N(t)$; however, there are only four independent equations (5-8) in five unknowns viz., $R_{i}(t)$, $i=1,2,3, \quad \rho(t)$ and $N(t)$. As such the system does not seem to have a unique solution. To obtain a determinate solution we require one additional assumption. Hence, we have considered two different assumptions:

i. In the particle creation scenario, the universe may start expanding with random fluctuations which may lead to different regions of the universe evolving in entirely different ways depending upon the mode of particle creation in those regions. In this sense, $N(t)$ may be regarded as an initial condition in such models. In the particle creation scenario, the entire behavior of the model including its expansion, deceleration/acceleration, entropy content etc., depends essentially upon the functional form of the particle creation function $N(t)$ i.e., the total number of particles as a function of time. Hence, by choosing the particle creation function $N(t)$, ab initio, the system of equations (5-8) would become well-defined and unique solutions can be obtained.

ii. In the second model the additional assumption is with respect to the average scale factor in terms of the deceleration parameter. We have considered the deceleration parameter to be a constant, that is

$$
q=\frac{-R \ddot{R}}{\cdot}=\beta
$$$$
(R)^{2}
$$

where $\beta$ is a constant and the average scale factor $R$ is given by $R=\left(R_{1} R_{2} R_{3}\right)^{1 / 3}$. Equation (13) can be rewritten as

$$
\frac{\ddot{R}}{R}+\beta\left(\frac{\dot{R}}{R}\right)^{2}=0
$$

On integration, equation (14) yields the exact solution

$R(t)=\left\{\begin{array}{cc}(D+C t)^{1 /(1+\beta)} & \beta \neq-1 \\ R_{0} e^{H_{0} t} & \beta=-1\end{array}\right.$

where $C, D, R_{0}$ and $H_{0}$ are constants of integration. In this paper we restrict our 
discussion to the power law expansion given by (15a). Using the expression for the average scale factor given by (15a) we obtain the expression for the particle creation function $N(t)$, energy density function and the particle creation pressure. We study the behaviour of the model for accelerated and decelerated expansions of the universe.

\section{Non-singular Inflationary Model with Expansion Driven by Creation of Matter}

In this section we discuss the cosmological model obtained for a particular choice of the particle creation fundtion.

In any open thermodynamical system, since volume is an extensive property, i.e., $V \propto N$, we have

$$
\frac{\dot{S}}{S}=\frac{\dot{N}}{N} \propto \frac{\dot{V}}{V}
$$

where $\frac{\dot{V}}{V}=\frac{\dot{R}_{1}}{R_{1}}+\frac{\dot{R}_{2}}{R_{2}}+\frac{\dot{R}_{3}}{R_{3}}$.

By considering the particle creation function to be determined by $\frac{\dot{N}}{N}=\frac{\dot{R}_{1}}{R_{1}}+\frac{\dot{R}_{2}}{R_{2}}+\frac{\dot{R}_{3}}{R_{3}}$, we see that equation (11) reduces to

$$
p_{c}=-(1+\gamma) \rho
$$

Using (17) in (9), we get

$$
\dot{\rho}=0, \text { i.e. } \rho=\rho_{c} \text { a constant. }
$$

Using (4), (17) and (18) in (5-8) yields

$$
\begin{aligned}
& \frac{\dot{R}_{1} \dot{R}_{2}}{R_{1} R_{2}}+\frac{\dot{R}_{1} \dot{R}_{3}}{R_{1} R_{3}}+\frac{\dot{R}_{2} \dot{R}_{3}}{R_{2} R_{3}}=8 \pi G \rho_{c} \\
& 2 \frac{\ddot{R}_{1}}{R_{1}}+\frac{\dot{R}_{1} \dot{R}_{2}}{R_{1} R_{2}}+\frac{\dot{R}_{1} \dot{R}_{3}}{R_{1} R_{3}}-\frac{\dot{R}_{2} \dot{R}_{3}}{R_{2} R_{3}}=8 \pi G \rho_{c} \\
& 2 \frac{\ddot{R}_{2}}{R_{2}}+\frac{\dot{R}_{1} \dot{R}_{2}}{R_{1} R_{2}}+\frac{\dot{R}_{2} \dot{R}_{3}}{R_{2} R_{3}}-\frac{\dot{R}_{1} \dot{R}_{3}}{R_{1} R_{3}}=8 \pi G \rho_{c} \\
& 2 \frac{\ddot{R}_{3}}{R_{3}}+\frac{\dot{R}_{1} \dot{R}_{3}}{R_{1} R_{3}}+\frac{\dot{R}_{2} \dot{R}_{3}}{R_{2} R_{3}}-\frac{\dot{R}_{1} \dot{R}_{2}}{R_{1} R_{2}}=8 \pi G \rho_{c}
\end{aligned}
$$

Adding (20), (21) and (22) leads to

$$
2 \frac{\ddot{R}_{1}}{R_{1}}+2 \frac{\ddot{R}_{2}}{R_{2}}+2 \frac{\ddot{R}_{3}}{R_{3}}+\frac{\dot{R}_{1} \dot{R}_{2}}{R_{1} R_{2}}+\frac{\dot{R}_{1} \dot{R}_{3}}{R_{1} R_{3}}+\frac{\dot{R}_{2} \dot{R}_{3}}{R_{2} R_{3}}=24 \pi G \rho_{c}
$$

Using (19) in (23) we get

$\frac{\ddot{R}_{1}}{R_{1}}+\frac{\ddot{R}_{2}}{R_{2}}+\frac{\ddot{R}_{3}}{R_{3}}=8 \pi G \rho_{c}$

From $V=R_{1} R_{2} R_{3}=R^{3}(t)$ we have

$\frac{\left(R^{3}(t)\right)}{R^{3}(t)}=\frac{\ddot{R}_{1}}{R_{1}}+\frac{\ddot{R}_{2}}{R_{2}}+\frac{\ddot{R}_{3}}{R_{3}}+2 \frac{\dot{R}_{1} \dot{R}_{2}}{R_{1} R_{2}}+2 \frac{\dot{R}_{1} \dot{R}_{3}}{R_{1} R_{3}}+2 \frac{\dot{R}_{2} \dot{R}_{3}}{R_{2} R_{3}}$

Using (19) in (25) and simplifying we get

$$
\frac{\ddot{R}_{1}}{R_{1}}+\frac{\ddot{R}_{2}}{R_{2}}+\frac{\ddot{R}_{3}}{R_{3}}=\frac{\left(R^{3}(t)\right)}{R^{3}(t)}-16 \pi G \rho_{c}
$$

From (24) and (26) we have

$$
\frac{\left(R^{3}(t) \ddot{)}\right.}{R^{3}(t)}=24 \pi G \rho_{c}=K
$$

where $K$ is a constant.

On integrating (27) we get

$$
R^{3}(t)=R^{3}(0) \exp \left(\sqrt{24 \pi G \rho_{c}} t\right)
$$

Adding (19) and (20) and simplifying leads to

$$
\frac{\ddot{R}_{1}}{R_{1}}+\frac{\dot{R}_{1} \dot{R}_{2}}{R_{1} R_{2}}+\frac{\dot{R}_{1} \dot{R}_{3}}{R_{1} R_{3}}=8 \pi G \rho_{c}
$$

The above equation can be rewritten as

$$
\frac{d\left(\dot{R}_{1} R_{2} R_{3}\right)}{d t}=8 \pi G \rho_{c} R^{3}
$$

Using (28) in (29) and integrating yields

$$
\frac{\dot{R}_{1}}{R_{1}}=\sqrt{\frac{8 \pi G \rho_{c}}{3}}+C_{1} \exp \left(-\sqrt{24 \pi G \rho_{c}} t\right)
$$

where $C_{1}$ is the constant of integration. For large times $t$, we see that the second term in (30) becomes very insignificant and it reduces to

$$
\frac{\dot{R}_{1}}{R_{1}}=\sqrt{\frac{8 \pi G \rho_{c}}{3}}
$$

On integrating (31) we get

$$
R_{1}(t)=R_{1}(0) \exp \left(\sqrt{\frac{8 \pi G \rho_{c}}{3} t}\right)
$$

Similarly, we get 


$$
\begin{aligned}
& R_{2}(t)=R_{2}(0) \exp \left(\sqrt{\frac{8 \pi G \rho_{c}}{3}} t\right) \\
& R_{3}(t)=R_{3}(0) \exp \left(\sqrt{\frac{8 \pi G \rho_{c}}{3} t}\right)
\end{aligned}
$$

Using the solutions for the scale factors given by (32), (33) and (34), we find the physical quantities of interest, the expansion scalar $\theta$, shear scalar $\sigma^{2}$, mean anisotropy parameter $A$ and deceleration parameter $q$ as follows:

$$
\begin{gathered}
\theta=3 H=\frac{\dot{R}_{1}}{R_{1}}+\frac{\dot{R}_{2}}{R_{2}}+\frac{\dot{R}_{3}}{R_{3}}=\sqrt{24 \pi G \rho_{c}} \\
\sigma^{2}=\frac{3}{2} A H^{2}=0 \\
A=\frac{1}{3} \sum_{i=1}^{3}\left(\frac{\Delta H_{i}}{H}\right)^{2}=0 \\
q=-1-\frac{\dot{H}}{H^{2}}=-1
\end{gathered}
$$

On integrating,

$$
\frac{\dot{N}}{N}=\frac{\dot{R}_{1}}{R_{1}}+\frac{\dot{R}_{2}}{R_{2}}+\frac{\dot{R}_{3}}{R_{3}}=\sqrt{24 \pi G \rho_{c}}
$$

we determine the total number of particles as

$$
N(t)=N(0) \exp \left(\sqrt{24 \pi G \rho_{c}} t\right)
$$

where $N(0)$ is the initial number of particles in the universe.

\section{Exact Solutions with Constant Deceleration Parameter}

In this section we discuss the cosmological model obtained by considering the deceleration parameter to be a constant.

Considering expression (15a) for the average scale factor, we get

$$
R^{3}(t)=(D+C t)^{3 /(1+\beta)}
$$

and hence, without loss of generality we can take

$$
\begin{aligned}
& R_{1}(t)=(D+C t)^{\alpha /(1+\beta)} \\
& R_{2}(t)=(D+C t)^{2 \alpha /(1+\beta)} \\
& R_{3}(t)=(D+C t)^{3 \alpha /(1+\beta)}
\end{aligned}
$$

From $(35-38)$ we get, $\alpha=1 / 2$. Consequently, we have

$$
\begin{aligned}
& R_{1}(t)=(D+C t)^{1 / 2(1+\beta)} \\
& R_{2}(t)=(D+C t)^{1 /(1+\beta)} \\
& R_{3}(t)=(D+C t)^{3 / 2(1+\beta)}
\end{aligned}
$$

Adding (6), (7) and (8) leads to

$2 \frac{\ddot{R}_{1}}{R_{1}}+2 \frac{\ddot{R}_{2}}{R_{2}}+2 \frac{\ddot{R}_{3}}{R_{3}}+\frac{\dot{R}_{1} \dot{R}_{2}}{R_{1} R_{2}}+\frac{\dot{R}_{1} \dot{R}_{3}}{R_{1} R_{3}}+\frac{\dot{R}_{2} \dot{R}_{3}}{R_{2} R_{3}}=-24 \pi G\left(p+p_{c}\right)$

Using (4) and (12) in (42) we get

$$
2 \frac{\ddot{R}_{1}}{R_{1}}+2 \frac{\ddot{R}_{2}}{R_{2}}+2 \frac{\ddot{R}_{3}}{R_{3}}+\frac{\dot{R}_{1} \dot{R}_{2}}{R_{1} R_{2}}+\frac{\dot{R}_{1} \dot{R}_{3}}{R_{1} R_{3}}+\frac{\dot{R}_{2} \dot{R}_{3}}{R_{2} R_{3}}=-24 \pi G \rho\left(\gamma-(1+\gamma) \frac{\dot{N}}{N} \frac{1}{3 H}\right)
$$

Using the expressions for $R_{1}(t), R_{2}(t), R_{3}(t)$ from (39-41) in (43) and simplying we obtain

$$
\frac{\dot{N}}{N}=a H, a=\frac{13+11 \gamma-8(1+\beta)}{11(1+\gamma)}
$$

Equation (44) on integration yields

$$
N=N_{0} R^{a}
$$

Using (44) in (9) and simplifying yields

$\frac{\dot{\rho}}{\rho}=(a-3)(1+\gamma) \frac{\dot{R}}{R}$

On integrating equation (46), we have

$\rho=\rho_{0} R^{(a-3)(1+\gamma)}$

Using (15a) in (47) and (45) we get

$$
\begin{gathered}
\rho(t)=\rho_{0}(C+D t)^{-k_{1}}, k_{1}=\frac{2(14+2 \beta+11 \gamma)}{11(1+\beta)} \\
N(t)=N_{0}(D+C t)^{a /(1+\beta)}
\end{gathered}
$$

In these models the expansion is driven by the creation of particles. Also, from the above expression for $N(t)$, we see that if $a=0$, $N$ would remain constant throughout the evolution of the universe.

In an open thermodynamic system we have $\frac{\dot{S}}{S}=\frac{\dot{N}}{N}$ and hence, $a \geq 0$. From (44) we see that

$$
\beta \leq \frac{5+11 \gamma}{8}
$$


From the expression for $a$ in (44), we note that the universe would undergo power law expansion $((1+\beta)>0)$ if

$$
0 \leq a<\frac{13+11 \gamma}{11(1+\gamma)}
$$

Also, for values of $a$ in the range

$$
0<\frac{5+11 \gamma}{11(1+\gamma)}<a<\frac{13+11 \gamma}{11(1+\gamma)}
$$

the expansion driven by creation would be accelerating, i.e. $\beta<0$. The expansion would be decelerating, i.e. $\beta>0$ for values of $a$ in the range

$$
0<a<\frac{5+11 \gamma}{11(1+\gamma)}
$$

In the matter dominated era, where we have $\gamma=0$, the universe would undergo accelerated expansion for values of $a$ in the range

$$
\frac{5}{11}<a<\frac{13}{11}
$$

The following table gives the values of the deceleration parameter for a few values of $a$.

\begin{tabular}{|c|c|}
\hline$a$ & $\beta$ \\
\hline $6 / 11$ & $-1 / 8$ \\
\hline $7 / 11$ & $-2 / 8$ \\
\hline $8 / 11$ & $-3 / 8$ \\
\hline $9 / 11$ & $-4 / 8$ \\
\hline $10 / 11$ & $-5 / 8$ \\
\hline 1 & $-6 / 8$ \\
\hline
\end{tabular}
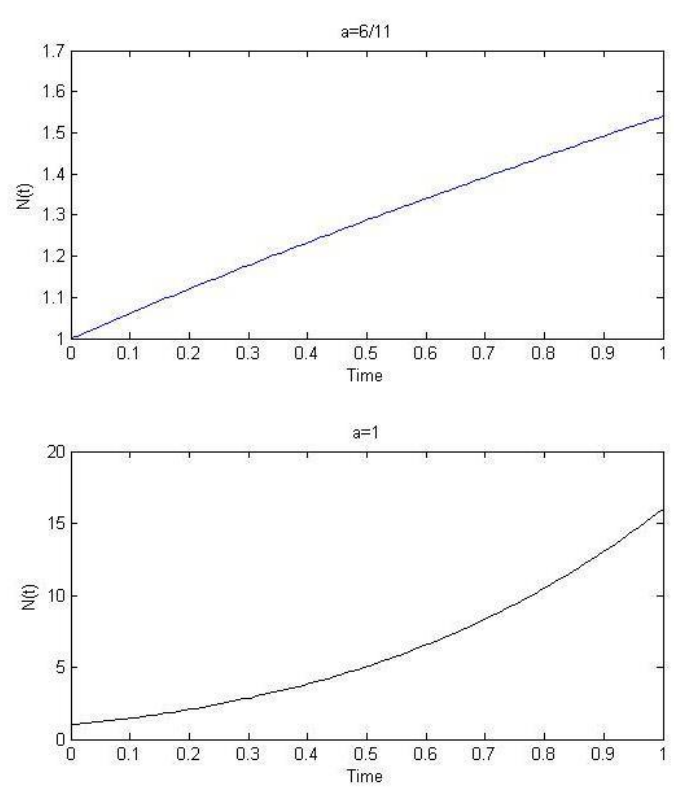

\section{\begin{tabular}{l|l}
$12 / 11$ & $-7 / 8$
\end{tabular}}

The following graph shows the relation between the deceleration parameter and ' $a$ ' in the matter dominated era.

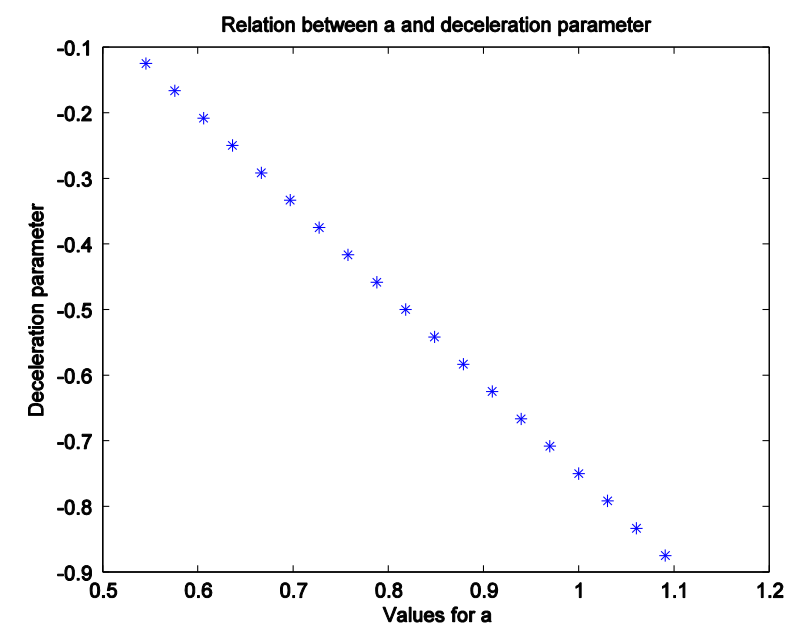

\section{Figure 1: Relation between 'a' and deceleration} parameter for matter dominated era

From Figure 1, we note that the expansion would be accelerating as seen from the negative values for the deceleration parameter. Figure 2 depicts the growth of the total number of particles $N(t)$ for 4 different values of 'a'.
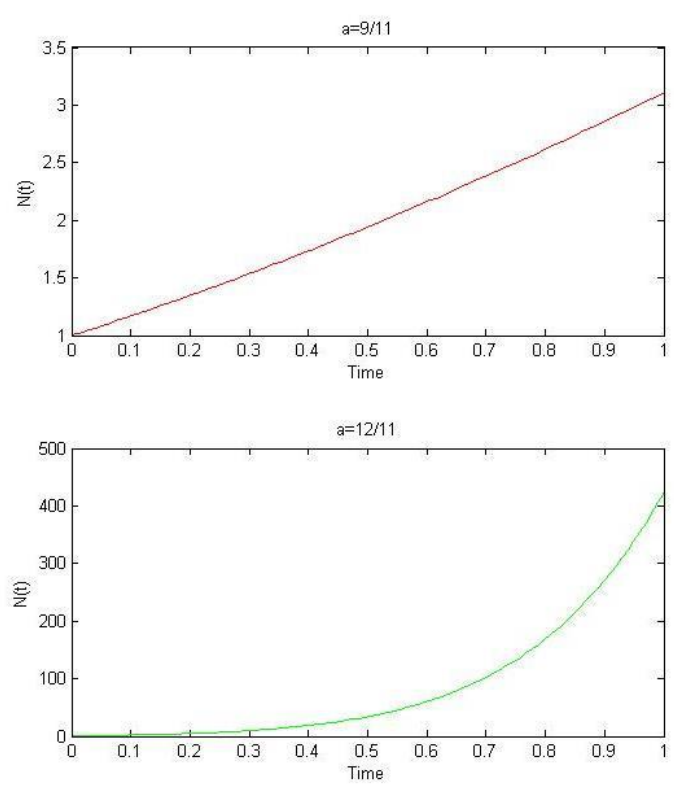

Figure 2: Behaviour of $N(t)$ for $a=6 / 11,9 / 11,1,12 / 11$ 
In Figures 3-5, we graphically depict the behaviour of the directional scale factors for 3 different values of the deceleration parameter.

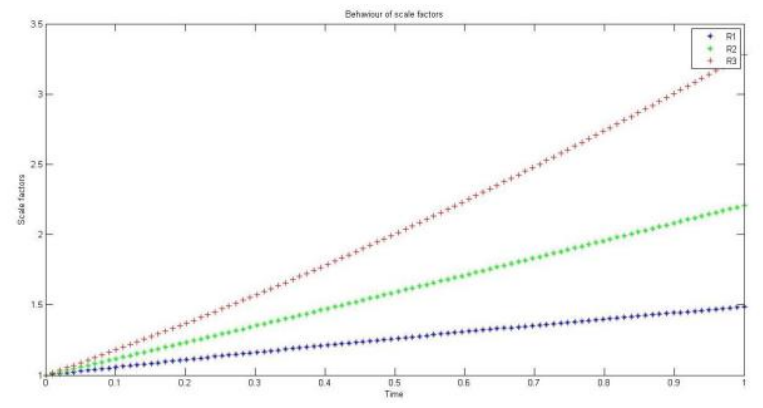

Figure 3: Behaviour of scale factors for deceleration parameter $=\mathbf{- 1 / 8}$

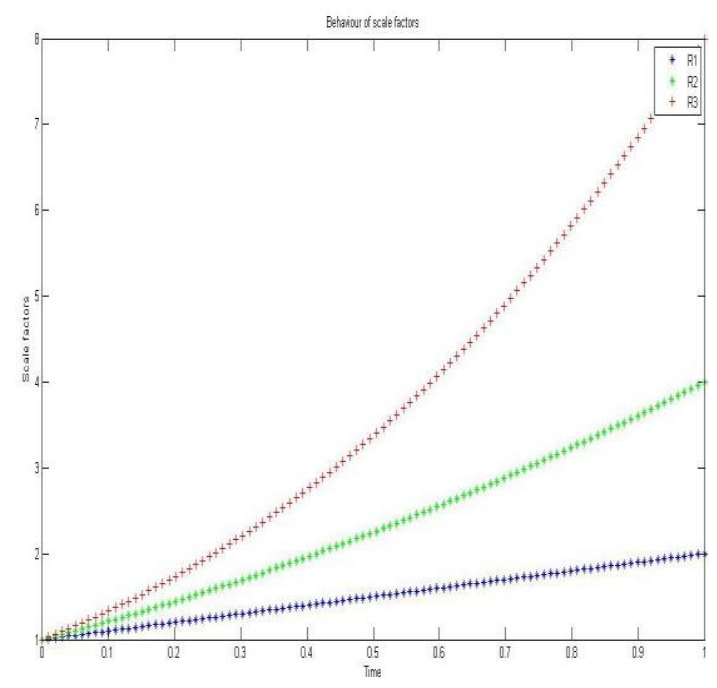

\section{REFERENCES}

1. Land, K., Magueijo, J. (2005). Phys. Rev. Lett. 95, 071301.

2. Bali, R., Singh, J.P. (2008). Int. J. Theor. Phys. 47, 3288.

3. Singh, J.P., Tiwari, R.K. (2008). Pramana 70, 565.

4. Singh, J.P., Tiwari, R.K., Prasad, A. (2008). Int. J. Theor. Phys. 47, 1559.

5. Krori, K.D., Mukherjee, A. (2000). Gen. Relativ. Gravit. 32, 1429.

6. Bhanja, S., Chakraborty, S., Debnath, U. (2005). Int. J. Mod. Phys. D. 14, 1919.

7. Riess A.G. et al. (1998). Astron. J.116, 1009.
Figure 4: Behaviour of scale factors for deceleration parameter $=-1 / 2$

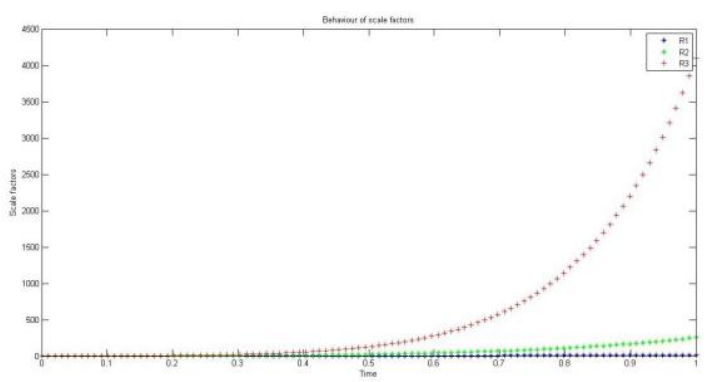

Figure 5: Behaviour of scale factors for deceleration parameter $=-7 / 8$

\section{CONCLUSION}

In this paper we have obtained solutions of the field equations of Bianchi type I anisotropic model of the universe in the presence of creation of matter. We have considered the universe as an open thermodynamic system. This gives rise to a negative pressure $p_{c}$ that drives the expansion of the universe.

For a particular choice of the particle creation function we have shown that the universe undergoes inflationary (exponential) expansion at late times. Due to inflationary expansion, the shear and anisotropy parameter become zero. The deceleration parameter takes the value -1 and the universe undergoes accelerated expansion.

We have also obtained the solution by considering the deceleration parameter to be a constant. The analysis shows that there is a wide variety of models with accelerated and decelerated expansions. We note that different choices for the three directional scale factors would lead to more interesting analysis.

8. Perlmutter S. et al, (1999). Astrophys. J.517, 565.

9. Padmanabhan T., (2003). Phys. Rept. 380, 235.

10. Peebles P.J.E., Ratra B., (2003). Rev. Mod. Phys. 75, 559.

11. Caldwell R.R., Dave R., Steinhardt P.J., (1998). Phys. Rev. Lett. 80, 1582.

12. Chiba T., Okabe T., Yamaguchi M., (2000). Phys. Rev. D 62, 023511.

13. Caldwell R.R., (2002). Phys. Lett. 545, 23.

14. Kamenshchik A.Y., Moschella U., Pasquier V., (2001). Phys. Lett. B 511, 265.

15. Carvalho J.C., Lima J.A.S., Waga I., (1992). Phys. Rev. D. 46, 2404. 
16. Wetterich C., (1995) Astron. Astrophys. 301, 321.

17. Arbab A.I., (1997). Gen. Relativ. Gravit. 29, 61.

18. Padmanabhan T., (2001). Preprint, $g r$ qc/0112068.

19. Vishwakarma R.G., (2002). Class. Quantum Gravity 19, 4747.

20. Shapiro I.L., Sola J., (2004). Preprint, astroph/0401015.

21. Dutta Choudhury S.B., Sil A., (2006). Astrophys. Space Sci. 301, 61.

22. Prigogine I., Geheniau J., Gunzig E. and Nardone P., (1988). Proc. Nat. Acad. Sci (USA), 85, 7428.

23. Prigogine I., Geheniau J., Gunzig E. and Nardone P., (1989). Gen. Relat. Grav. 21, 767.

24. Prigogine I., Geheniau J., (1986). Proc. Nat. Acad. Sci (USA) 83, 6245.

25. Prigogine I. and Glansdorff J., (1971) Thermodynamic Theory of Structure, Stability and Fluctuations, Wiley Interscience, New York.

26. Calvaao, M. O., Lima, J. A. S., and Waga, I. (1992). Phys. Lett. A 162, 223.

27. Sudharsan, R., and Johri, V. B. (1994). Gen. Rel. Grav. 26, 41.

28. Desikan, K. (1997). Gen. Rel. Grav. 29, 435.

29. Lima, J. A. S., Germano, A. S. M., and Abramo, L. R. W. (1996). Phys. Rev. D53, 4287.

30. Abramo, L. R. W., and Lima, J. A. S. (1996). Class. Quantum Grav. 13, 2953.

31. Lima, J. A. S. (1997). Gen. Rel. Grav. 29, 805.

32. Johri V.B. and Desikan K., (1994). Gen. Relat. Grav., 26, 1217.

33. Singh, G.P., Beesham, A. (1999). Aust. J. Phys. 52, 1039.

34. Singh, G.P., Deshpande, R.V., Singh, T. (2002). Astrophys. Space Sci. 282, 489.

35. Singh, G.P., Beesham, A., Deshpande, R.V. (2000). Pramana 54, 729.

36. Harko, T., and Mak, M. K. (1997). Astrophys. Space Sci. 253, 161.

37. Brevik, I., and Stokkan, G. (1996). Astrophys. Space Sci. 239, 89.

38. Gunzig, E., Maartens, R., and Nesteruk, A. V. (1998). Class. Quantum Grav. 15, 923.

39. Zimdahl, W., and Pav'on, D. (1993). Phys. Lett. A176, 57.

40. Zimdahl, W., Pav'on, D., and Jou, D. (1993). Class. Quantum Grav. 10, 1775.

41. Zimdahl, W., and Pav'on, D. (1994). Int. J. Mod. Phys. D3, 327.

42. Zimdahl, W., and Pav'on, D. (1994). Gen. Rel. Grav. 26, 1259.

43. Bali, R. (2011). Int. J. Theor. Phys. 50, 3043.

44. Bali, R., Singh, J.P. (2008). Int. J. Theor. Phys. 47, 3288.

45. Chimento, L.P., Jakubi, A.S., Mendez, W., Maartens, R. (1997). Class. Quantum Gravity 14, 3363.
46. Pradhan, A., Pandey, P. (2006). Astrophys. Space Sci. 301, 127.

47. Pradhan, A., Singh, S.K. (2004). Int. J. Mod. Phys. D 13, 503.

48. Yadav, A.K., Saha, B. (2011). Astrophys. Space Sci. doi:10.1007/s10509-011-0861-0.

49. Harko, T., and Mak, M. K. (2000). Gen. Rel. Grav. 32, 865.

50. Singh, G.P., Kale, A.Y. (2011). Astrophys. Space Sci. 331, 207. 